
153.

(10) Samdal, S.; Seip, H. M.; Torgrimsen, T. J. Mol. Struct. 1977, 42,

(11) Schei, S. H.; Seip, R. Acta Chem. Scand. 1984, A38, 345.

(12) (a) Keyaniyan, S.; Göthling, W.; de Meijere, A. Chem. Ber. 1984, 120,395 and references therein. (b) Keyaniyan, S.; Göthling, W.; de Meijere, A. Tetrahedron Lett. 1984, 25, 4105 .

(13) Bastiansen, O.; Hassel, O.; Risberg, E. Acta Chem. Scand. 1955, 9, 232.

(14) Hagen, K.; Hedberg, K. J. Am. Chem. Soc. 1973, 95, 1003

(15) Gundersen, G.; Hedberg, K. J. Chem. Phys. 1969, 51, 2500.
(16) Andersen, B.; Seip, H. M.; Strand, T.; Stølevik, R. Acta Chem Scand. 1969, 23, 3224.

(17) Hedberg, L. Abstracts of Papers, 5th Austin Symposium on GasPhase Molecular Structure, Austin, TX, March 1974; p 37.

(18) International Tables of X-ray Crystallography; Vol. III, to be published.

(19) Hedberg, K.: Iwasaki, M. Acta Crystallogr. 1964, 17, 529.

(20) Klaeboc, P.; Neerland, G.; Schei, S. H. Spectrochim. Acta 1982, $338 \mathrm{~A}, 1025$.

(21) Kaleem, H.: Stølevik, R.; Hagen, K., to be published.

\title{
Wall-Jet Electrode LInear Sweep Voltammetry
}

\author{
Richard G. Compton, ${ }^{*}$ Adrian C. Fisher, Mark H. Latham, \\ Physical Chemistry Laboratory, South Parks Road, Oxford OXI 3QZ, United Kingdom \\ Christopher M. A. Brett, and Ana Maria C. F. Oliveira Brett \\ Departamento de Quimica, Universidade de Coimbra, 3049 Coimbra, Portugal (Received: July 31, 1991)
}

\begin{abstract}
Theory is presented which predicts the linear sweep voltammetry behavior at the wall-jet electrode for a reversible couple. The scan rate and electrode geometry dependences are established, and hence the requirements for the measurement of true "steady state" hydrodynamic voltammograms are defined. Theory is found to be in good agreement with experiments conducted on the oxidation of the ferrocyanide anion in aqueous solution.
\end{abstract}

\section{Introduction}

The wall-jet electrode (WJE) is a well-characterized hydrodynamic electrode in which the flow is due to a (submerged) fluid jet which strikes a planar electrode at right angles and spreads out radially over that surface, the fluid outside the jet being at rest. ${ }^{1}$ The mass transport experienced by the electrode is dependent on its size relative to the impinging jet, and two extremes are recognized. The term "wall jet" is understood to pertain to the case where the electrode is substantially larger than the jet, ${ }^{1-6}$ whereas the other limit, in which the relatively tiny electrode is within a stagnant flow region, is defined as of "wall-tube" geometry. ${ }^{7}$ The description "impinging jet" finds occasional usage and may relate to either the wall jet or wall tube. ${ }^{8-10}$ In this paper we are concerned with the wall jet as described above and the mass transport characteristics of this system are outlined below. ${ }^{1-6}$

WJEs are finding increasing use in analysis, e.g. ref 11 , primarily due to the advantages of on-line detection and fast sample throughput. Moreover, in the context of the mechanistic investigation of electrode processes, the wall-jet geometry has been shown to possess considerable advantages most notably due to its highly nonuniform primary current distribution. ${ }^{12}$ Further merits arise first from the flow-through nature of the device which means that the constant supply of fresh solution prevents the buildup of intermediates and products of the electrode reaction which might otherwise alter the course of the electrode process (chemostatic conditions) and second from the high sensitivity of the wall jet (as compared to, say, the rotating disc electrode) to variations in the rate of mass transport. The use of wall-jet electrodes in flow analysis has been recently reviewed. ${ }^{13}$

In this paper we establish theory which predicts the current/voltage response resulting from a potential sweep, at the wall-jet electrode. The problem is of interest since it effectively defines the "response time" of the electrode and, additionally, such simulations can define the range of scan rates for which an effective steady-state current/voltage curve can be recorded. Experiments are reported which quantitatively confirm the theoretical predictions.

\section{Theory \\ We examine a simple reversible one electron transfer}

$$
A \pm \mathrm{e}^{-} \rightleftharpoons \mathrm{B}
$$

and consider the case where the electrode potential is swept from a potential at which no current flows to one that corresponds to the transport limited reduction/oxidation of species $\mathbf{A}$. The convective diffusion equation describing the concentration of $\mathbf{A}$ in time $(t)$ and space is

$$
\frac{\partial[\mathrm{A}]}{\partial t}=D \frac{\partial^{2}[\mathrm{~A}]}{\partial z^{2}}-v_{r} \frac{\partial[\mathrm{A}]}{\partial r}-v_{z} \frac{\partial[\mathrm{A}]}{\partial z}
$$

where $D$ is the diffusion coefficient of $\mathrm{A}, v_{\mathrm{r}}$ is the radial solution velocity ( $r$ direction), and $v_{z}$ is the velocity in the direction normal to the electrode surface ( $z$ direction). Expressions for $v_{r}$ and $v_{z}$ for wall-jet flow are given in ref 12 . Note that in writing eq 1 radial diffusion has been neglected: the basis of this approximation has been developed elsewhere. ${ }^{14}$ We also assume the presence of sufficient supporting electrolyte that migration effects are negligible.

The electrode potential $E_{t}$ is swept linearly with time at a rate $v \mathrm{~V} \mathrm{~s}^{-1}$ (where $v$ is either positive or negative) through the reduction/oxidation wave of $\mathrm{A}$, starting from an initial potential $E_{\text {l. }}$.

$$
E_{\mathrm{t}}=E_{1}-v t
$$

The relevant boundary conditions to the problem, as defined above, may be formulated as

$$
\begin{aligned}
& \tau=0, z \geq 0,0<r<R \quad[\mathrm{~A}]=[\mathrm{A}]_{\text {bulk }} \quad[\mathrm{B}]=0 \\
& \tau>0, z=0,0<r<R \quad \frac{\partial[\mathrm{A}]}{\partial z}=-\frac{\partial[\mathrm{B}]}{\partial z} \\
& \tau>0, z=0,0<r<R \quad \frac{[\mathrm{A}]}{[\mathrm{B}]}=\theta \exp (-\sigma \tau) \\
& \text { all } \tau, z \rightarrow \infty, 0<r<R \quad[\mathrm{~A}]=[\mathrm{A}]_{\text {bulk }} \quad[\mathrm{B}]=0
\end{aligned}
$$

where $[A]_{\text {bulk }}$ is the bulk concentration of $A$,

$$
\begin{gathered}
\theta=\exp \left[\left(\frac{e}{k_{\mathrm{B}} T}\right)\left(E_{\mathrm{I}}-E^{\ominus}\right)\right] \\
\tau=\left(\frac{A^{2} D}{R^{14 / 8}}\right) t \\
\sigma=\left(\frac{e v}{k_{\mathrm{B}} T}\right)\left(\frac{R^{14 / 8}}{A^{2} D}\right)
\end{gathered}
$$


$\mathrm{A}=R^{-3 / 8}(9 C / 8 D)^{1 / 3}, C=\left\{[5 M]^{3} / 216 v^{5}\right\}^{1 / 4}, M=k_{\mathrm{c}}^{4} V_{\mathrm{f}}^{3} / 2 \pi^{3} a^{2}$, $e$ is the electronic charge, $k_{\mathrm{B}}$ is Boltzmann's constant $\left(e / k_{\mathrm{B}} T\right)=$ $38.9 \mathrm{~V}^{-1}$ at $25^{\circ} \mathrm{C}$ ), $v$ is the kinematic viscosity of the solution, and $R$ is the radius of the electrode. The reason for defining $\tau$ (normalized time) and $\sigma$ (dimensionless scan rate) as given is explained in the Appendix. In eq 3 we have made the approximation that $A$ and $B$ have identical diffusion coefficients.

In order to solve eq 1 we approximate the derivatives by their finite-difference equivalents. The $r-z$ plane is thus divided up into a two-dimensional grid such that increments in the $r$ direction are $\Delta \boldsymbol{r}$ and in the $z$ direction $\Delta z$. We use the subscripts $k$ and $j$ to denote distances in the radial and normal directions:

$$
\begin{gathered}
\text { (radial distance) })_{k}=k \Delta r \\
k=0,1,2, \ldots, K, \text { where } \Delta r=R / K \\
(\text { normal distance })_{i}=j \Delta z \\
j=0,1,2, \ldots, J, \text { where } \Delta z=Z / J
\end{gathered}
$$

and $Z$ is the length of the grid in the $z$ direction. ${ }^{12}$ Then the notation ${ }^{t} a_{j, k}$ indicates the concentration of $\mathrm{A}$ at the point $(j, k)$ at the instant $t \Delta t$, where $\Delta t$ is a selected increment of real time (say, for example, $0.1 \mathrm{~ms}$ ).

The finite-difference form of eq 1 is

$$
\begin{aligned}
{ }^{t+1} a_{j, k}-{ }^{t} a_{j, k}= & \lambda^{y}\left\{t^{t+1} a_{j+1, k}-2^{t+1} a_{j, k}+{ }^{t+1} a_{j-1, k}\right\}- \\
& \left.\left.\left.\lambda_{j,\{} t^{t+1} a_{j, k}-{ }^{t+1} a_{j, k-1}\right\}-\lambda_{j}\right\}^{v_{j}} t^{t+1} a_{j+1, k}-{ }^{t+1} a_{j, k}\right\}
\end{aligned}
$$

where

$$
\begin{gathered}
\lambda^{y}=\frac{D \Delta t}{(\Delta z)^{2}} \\
\lambda_{j}^{v_{r}}=\frac{v_{r}(j+1, k) \Delta t}{\Delta r} \\
\lambda_{j}^{v_{z}}=\frac{v_{z}(j+1, k) \Delta t}{\Delta z}
\end{gathered}
$$

and $v_{r}(j, k)$ and $v_{z}(j, k)$ are the solution velocity components at $(j, k)$. Rearrangement of eq 13 leads to the following general equation used as the basis for the ensuing (implicit) calculations:

$$
\begin{aligned}
& \left.\left.\left\{a_{j, k}\right\}+\lambda_{j}{ }^{p_{2}\{t+1} a_{j, k-1}\right\}=-\left.\lambda^{y}\right|^{t+1} a_{j-1, k}\right\}+
\end{aligned}
$$

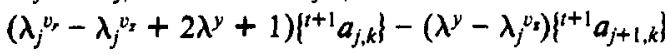

Application of the boundary condition (4) leads to

$$
\begin{aligned}
& \left.\left\{a_{1, k}\right\}+\lambda_{1}^{v_{r} \mid t+1} a_{1, k-1}\right\}+\frac{\lambda^{y} \theta \exp (-\sigma(t+1) \Delta \tau)}{1+\theta \exp (-\sigma(t+1) \Delta \tau)}= \\
& \left.\left.\left.\left.\left(\lambda_{l}^{v_{r}}-\lambda_{l}^{v_{z}}+2 \lambda^{y}+1\right)\right\}^{t+1} a_{1, k}\right\}-\left(\lambda^{y}-\lambda_{l}^{y_{z}}\right)\right\}^{t+1} a_{2, k}\right\}
\end{aligned}
$$

and condition (5) to

$$
\begin{aligned}
& \left.\left.\left\{a_{J-1, k}\right\}+\lambda_{J-1}{ }^{v_{r}}\left\{t^{t+1} a_{J-1, k-1}\right\}=-\lambda^{y}\right\}^{t+1} a_{J-2, k}\right\}+ \\
& \left.\left.\left(\lambda_{-1}^{J} v_{r}-\lambda_{J-1}^{v_{z}}+2 \lambda^{y}+1\right)\right|^{t+1} a_{J-1, k}\right\}-\left(\lambda^{y}-\lambda_{J-1} v_{z}\right)
\end{aligned}
$$

These $(J-1) \times(J-1)$ simultaneous equations may be expressed as a $(J-1) \times(J-1)$ matrix equation:

$$
\{\mathbf{d}\}=[\mathbf{T}]\{\mathbf{u}\}
$$

where

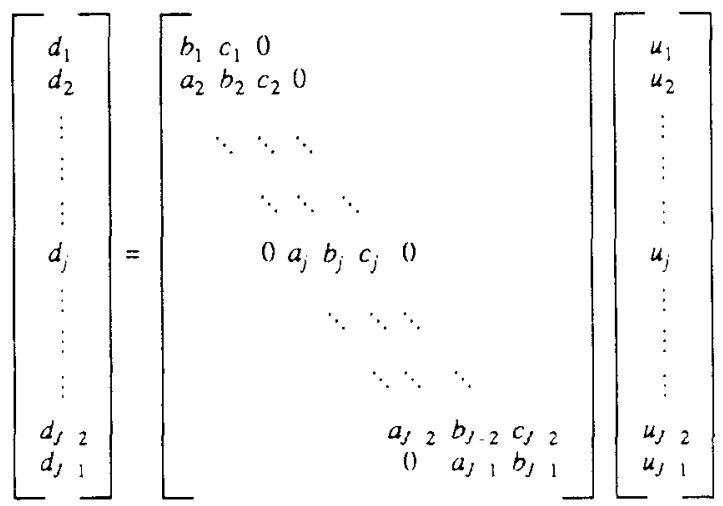

The matrix elements are given by

$k=1$

$$
\begin{gathered}
d_{1}=\left\{a_{1,1}\right\}+\lambda_{1}{ }^{{ }^{r}}+\frac{\lambda^{y} \theta \exp (-\sigma(t+1) \Delta \tau)}{1+\theta \exp (-\sigma(t+1) \Delta \tau)} \\
d_{j}=\left\{a_{j, 1}\right\}+\lambda_{j}{ }^{v_{r}} \quad j=2,3, \ldots, J-2 \\
d_{J-1}=\left\{a_{J-1, k}\right\}+\lambda_{J-1}{ }^{v_{r}}+\left(\lambda^{y}-\lambda_{J-1}^{v_{z}}\right)
\end{gathered}
$$

$k>1$

$$
\begin{array}{r}
\left.d_{l}=\left\{a_{1,1}\right\}+\lambda_{1}^{v_{f}\{t+1} a_{1, k-1}\right\}+\frac{\lambda^{y} \theta \exp (-\sigma(t+1) \Delta \tau)}{1+\theta \exp (-\sigma(t+1) \Delta \tau)} \\
\left.d_{j}=\left\{a_{j, k}\right\}+\lambda_{j}^{v_{r}\{t+1} a_{j, k-1}\right\} \quad j=2,3, \ldots, J-2 \\
\left.d_{J-1}=\left\{a_{J-1, k}\right\}+\lambda_{J-1}{ }^{v_{f}\{t+1} a_{J-1, k-1}\right\}+\left(\lambda^{y}-\lambda_{J-1}^{v_{z}}\right)
\end{array}
$$

all $k$

$$
\begin{aligned}
& a=-\lambda^{y} \\
& b_{j}=2 \lambda^{y}+\lambda_{j}^{v_{r}}-\lambda_{j}^{v_{z}}+1 \quad j=1,2, \ldots, J-1 \\
& c_{j}=-\left(\lambda^{y}-\lambda_{j}^{v_{z}}\right) \quad j=1,2, \ldots, J-1
\end{aligned}
$$

Equation 20 is analogous to that arising in the procedure we have previously described ${ }^{12}$ for the solution of steady-state problems at the WJE and we have adopted here the same notation as in that paper. The method of solution below follows the general strategy developed in ref 12 . A brief summary is given in the next paragraph.

Notice that the matrix equation (20) shows how the concentrations throughout the cell at time $(t+1) \Delta t$ may be calculated if we know those at time $t \Delta t$. To do this we have to find the set of vectors $\{u\}$ : each $k$ value has its own vector $\{a\}_{k}$. The matrix [T] being of tridiagonal form allows us to use the Thomas algorithm $^{12,15}$ to give $\{u\}_{k}$ from $\{d\}_{k}$. The boundary condition (2) supplies the vector $\{d\}_{0}$ from which $\{u\}_{0}$ is calculated. Then $\{d\}_{k+1}$ $=\{u\}_{k}$, so $\{u\}_{1}$ is calculated from $\{d\}_{1}$, and so on until $\{u\}_{k}$ is obtained. The calculation is then repeated. Note that an expanding grid, which increases in size in proportion to the diffusion layer thickness, is used on account of the electrode being very nonuniformly accessible so as to give accurate results. The protocol for the implementation of this is exactly as in the solution of steady-state WJE problems. ${ }^{12}$

In this way the concentration profile of $A$ within the WJE may be calculated as a function of time. The current at the electrode may thus be evaluated at any instant from

$$
{ }^{t} I=2 \pi D F \sum_{k=1}^{K}[\mathrm{~A}]_{\text {bulk }}\left({ }^{t} a_{1, k}-{ }^{\prime} a_{0, k}\right) \frac{k(\Delta r)^{2}}{\Delta z}
$$

and thus the form of the current-voltage curve established.

\section{Theoretical Results and Discussion}

Using the theory outlined above, potential sweep transients were computed (using a Sun Sparc workstation); copies of the FORTRAN program are available on request from the authors. Convergence was examined by varying $J, K$, and $\Delta t$ values. For a typical electrode of geometry of radius $R=0.1637 \mathrm{~cm}$, cell constant $k_{\mathrm{c}}=0.9$, and jet diameter $a=0.0345 \mathrm{~cm}$, for volume solution flow rates in the range $0.1>V_{\mathrm{f}} / \mathrm{cm}^{3} \mathrm{~s}^{-1}>0.001$ values of $J=200, K=200$, and $\Delta t=0.001 \mathrm{~s}$ were found to give satisfactory and rapid ${ }^{15}$ convergence (to 3 significant figures) with typical aqueous solution parameters $\left(v=0.0089 \mathrm{~cm}^{2} \mathrm{~s}^{-1} ; 1 \times 10^{-6}\right.$ $<D / \mathrm{cm}^{2} \mathrm{~s}^{-1}<1 \times 10^{-5}$ ).

Initial computations were undertaken to test the predictions of the numerical approach at very slow scan rates and compared to the behavior computed directly for steady-state conditions from the appropriate boundary conditions. ${ }^{16}$ Figure 1 shows the current (normalized to the steady-state limiting current) response of a steady-state calculation for a reduction wave using a flow rate 


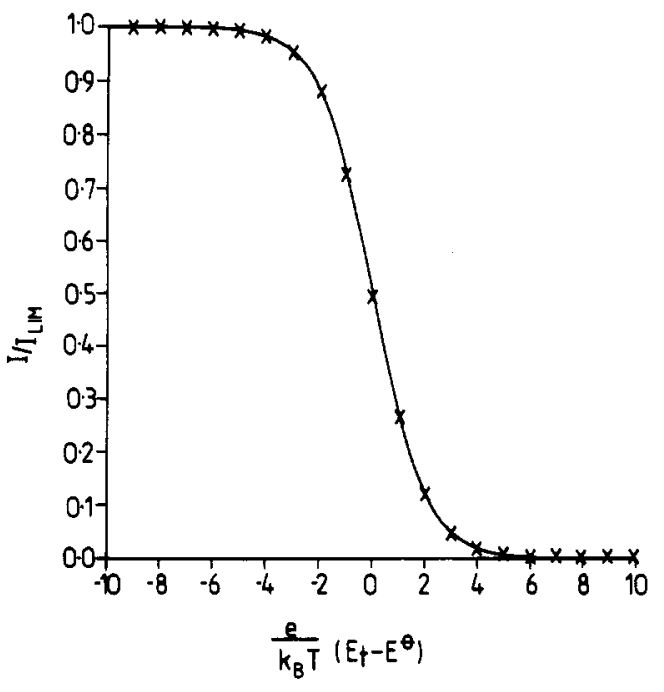

Figure 1. Slow scan rate voltammogram calculated for the conditions and parameters specified in the text calculated using the new transient theory described in this paper and, for comparison, the authentic steady-state behavior $(x)$ calculated using established theory. ${ }^{16}$

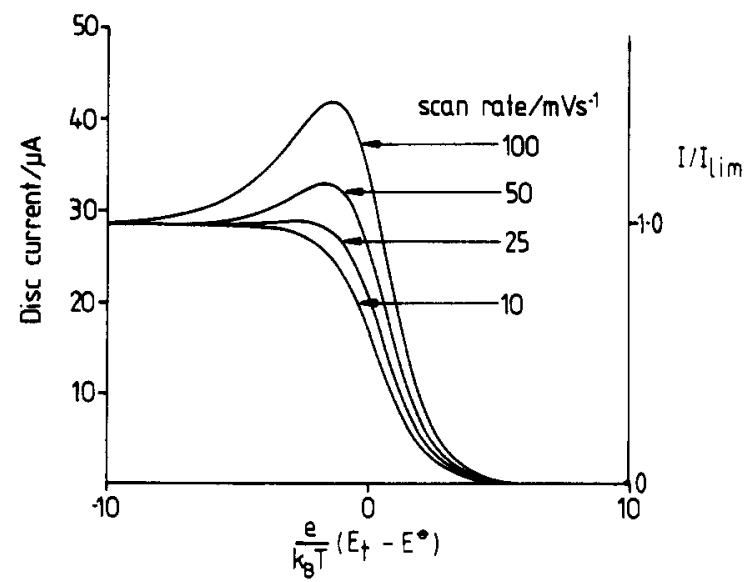

Figure 2. Current-voltage curves for computed for the scan rates 10, 25, 50 , and $100 \mathrm{mV} \mathrm{s}^{-1}$ for the electrode and parameters defined in the text. The values of $\sigma$ corresponding to these scan rates are $1.62,4.05,8.10$, and 16.20 (see text).

of $V_{\mathrm{f}}=0.1 \mathrm{~cm}^{3} \mathrm{~s}^{-1}$, for the electrode geometry specified earlier, with a scan rate of $0.001 \mathrm{~V} \mathrm{~s}^{-1}$. A diffusion coefficient of $5 x$ $10^{-6} \mathrm{~cm}^{2} \mathrm{~s}^{-1}$ was assumed. As can be seen, excellent agreement is observed, supporting our general theoretical approach.

Having established the validity of the method, we then applied the approach to predict the response of a current/voltage scan under increasingly faster. scan rates for a reduction process at an electrode with the wall-jet geometry detailed above. Figure 2 shows a typical plot for a flow rate of $0.95 \times 10^{-2} \mathrm{~cm}^{3} \mathrm{~s}^{-1}$, with the varying scan rates detailed. The following parameters were employed in the computations: $D=6.5 \times 10^{-6} \mathrm{~cm}^{2} \mathrm{~s}^{-1},[\mathrm{~A}]_{\text {bulk }}$ $=2 \times 10^{-6} \mathrm{~mol} \mathrm{~cm}^{-3}$. As can be seen, with increasing scan rate finite diffusion in solution causes the concentration profile of $A$ to "lag behind" that of the steady-state response and a peak appears in the current/voltage curve. At the same time, the wave becomes shifted anodically from that observed under steady-state conditions. As indicated in the Appendix voltammograms such as those in Figure 2 are, if the current is normalized to the steady-state limiting current (as on the right hand $y$ axis of Figure 2 ), simply a unique function of $\sigma$ and $\theta$. Thus, the four curves shown are general in that they relate to any wall-jet system with the same $\sigma$ values as specified in the figure legend and to assist the reader in the application of our analysis to his experiments we present numerically the results plotted in Figure 2 in Table I.

Next, the simulations were employed to establish the criteria for the experimental measurement of steady-state voltammograms.
TABLE I

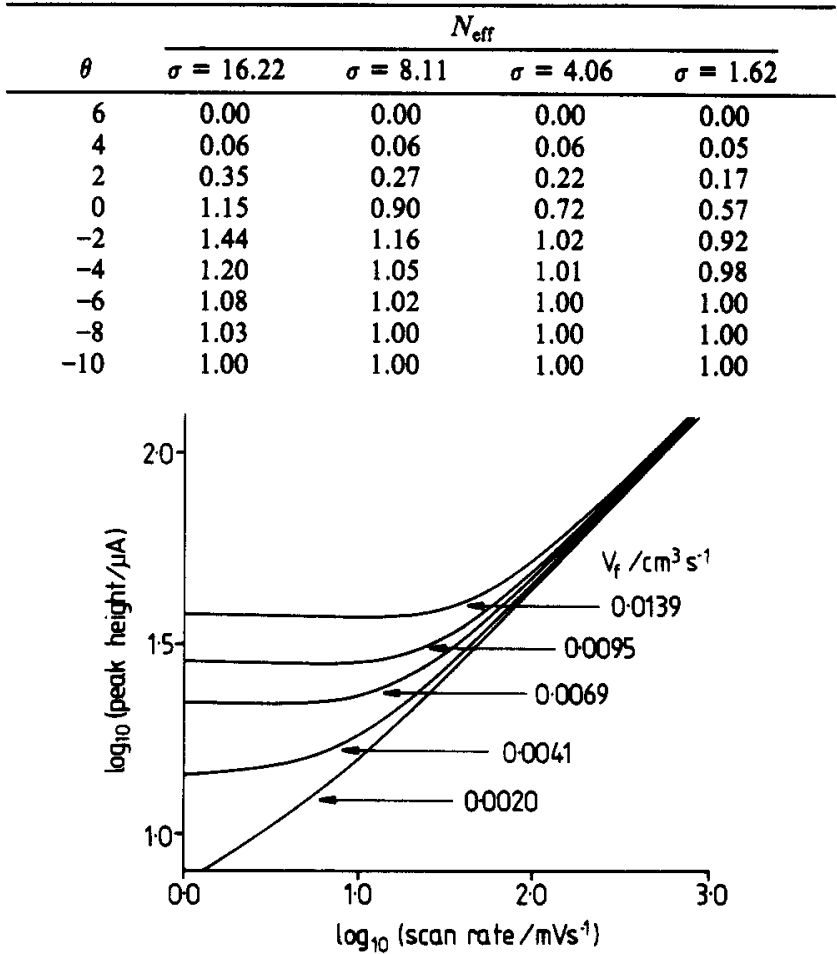

Figure 3. Dependence of the peak current on the scan rate for the electrode and parameters specified in the text.

A wide range of flow rates and scan rates were employed. The results are best presented by a plot of $\log$ (peak current) against $\log$ (scan rate) as given in Figure 3 for the same electrode geometry and values for $D$ and $[A]_{\text {bulk }}$ as were used to generate Figure 2 . At slow scan rates the maximum current observed is the normal steady-state limiting current. However, as the scan rate is increased a peak is observed as noted above and as the scan rate is increased even further this peak current tends to the behavior of a purely diffusional regime. From Figure 3 it can be seen at very fast scan rates a slope of $1 / 2$ is observed corresponding to this diffusional limit, whereas at slow scan rates the peak current is independent of scan rate. Plots such as Figure 3 define the range of scan rates for a particular flow rate at which authentic steady-state voltammograms may be obtained, for example, for Tafel, or other analysis. Specifically, generalizing from Figure 2 , steady-state voltammograms will result providing the parameter $\sigma$ is of order unity or below.

Finally, it should be pointed out that the results shown in Figure 3 may be independently checked in both the slow and fast scan rate limits. In the former case we find good agreement with analytical theory for steady-state currents at the $\mathrm{WJE}^{14}$ and in the latter our computations quantitatively reproduce the established theory for the cyclic voltammetry of a reversible couple in stationary solution [see, e.g., ref 17].

\section{Experimental Section}

Experiments to test the validity of the theory derived above were carried out using as model reaction the oxidation of potassium ferrocyanide at a wall-jet platinum disc electrode of radius 1.637 $\mathrm{mm}$ contained in a large volume wall-jet cell constructed of Perspex. The tip of a Radiometer K401 SCE reference electrode was placed in the flow stream just before the entry to the wall-jet cell. This arrangement was found, during the recording of steady-state voltammograms, to be the best in minimizing any effects of uncompensated ohmic drop, as effectively the reference electrode is located at the nozzle inlet (diameter $0.345 \mathrm{~mm}$ ) into the cell. The nozzle-electrode distance was $3 \mathrm{~mm}$ in all experiments. A platinum gauze auxiliary electrode was contained within the cell.

The test solution was $2.01 \mathrm{mM} \mathrm{K}_{4} \mathrm{Fe}(\mathrm{CN})_{6}$ in $0.4 \mathrm{M} \mathrm{K}_{2} \mathrm{SO}_{4}$ supporting electrolyte, prepared from analytical reagents and 


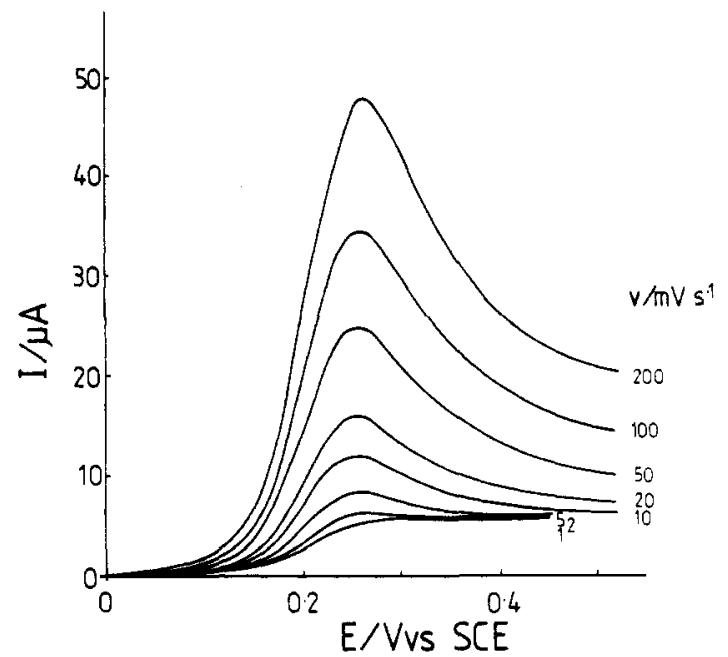

Figure 4. Typical set of voltammograms (solid lines) for different voltage scan rates $\left(1,2,5,10,20,50,100,200 \mathrm{mV} \mathrm{s}^{-1}\right.$ for the oxidation of 2.01 $\mathrm{mM} \mathrm{K} \mathrm{Fe}(\mathrm{CN})_{6}$ in $0.4 \mathrm{M} \mathrm{K}_{2} \mathrm{SO}_{4}$ measured at a flow rate of $V_{\mathrm{f}}=0.002$ $\mathrm{cm}^{3} \mathrm{~s}^{-1}$.

tridistilled water. A Pharmacia P-3 peristaltic pump was used to drive solution through the cell with a glass ball capacitor and $5 \mathrm{~m}$ length of $1 \mathrm{~mm}$ internal diameter Teflon tubing placed before the cell in order to minimize pump pulsation. Flow rates ranging from 0.002 to $0.051 \mathrm{~cm}^{3} \mathrm{~s}^{-1}$ were employed.

Experiments were conducted using a conventional style potentiostat (Oxford Electrodes) with scan rates varying from 1 to $500 \mathrm{mV} \mathrm{s}^{-1}$. Linear sweep voltammograms were registered on a Hewlett-Packard 7035B X-Y recorder. All experiments were carried out under thermostated conditions at $25 \pm 0.1^{\circ} \mathrm{C}$.

\section{Results and Discussion}

A typical set of voltammograms is presented in Figure 4 which shows the expected evolution of a voltammetric peak as the voltage scan rate is increased. From families of current/voltage curves such as those in Figure 4, a plot of $\log$ (peak current) vs $\log$ (scan rate), analogous to Figure 3, can be constructed and is shown in Figure 5. Also shown (Figure 5b) is the computed behavior for corresponding conditions deduced from the theory presented above using a diffusion coefficient of $6.5 \times 10^{-6} \mathrm{~cm}^{2} \mathrm{~s}^{-1}$ as deduced from steady-state measurements. Reasonable agreement between parts $a$ and $b$ of Figure 5 is apparent. In particular, the appearance of the peak at increasingly higher scan rates as the flow rate is increased is confirmed, as is the tendency to the diffusion-limited relationship of $d \log$ (peak current) $/ d \log (v)=0.5$ at high scan rate.

Figure 6 is a plot of the half-wave potential determined at 1 $\mathrm{mV} \mathrm{s}^{-1}$ scan rate for various flow rates. This plot is important-it shows that for flow rates less than $0.015 \mathrm{~cm}^{3} \mathrm{~s}^{-1}$, and with the electrode geometry selected, a true measurement of $E^{\ominus}$ from $E_{1 / 2}$ cannot be made unless the scan rate is reduced accordingly. In this context it should be noted that there is a negative shift in $E_{1 / 2}$ before the appearance of a peak in the voltammogram. Similar conclusions have been drawn in respect of analogous measurements made at the rotating disc electrode. ${ }^{18}$

\section{Conclusions}

We have deduced theoretically the linear sweep voltammetry response for a reversible electrode process at the wall-jet electrode. Experiment confirms the theoretical approach and gives good quantitative agreement. The requirement of using a sufficiently slow scan rate at a particular flow rate for the determination of the formal potential from the measured half-wave potential is defined.

\section{Appendix}

Consider eq 1 and note that with the explicit equations for $v_{r}$
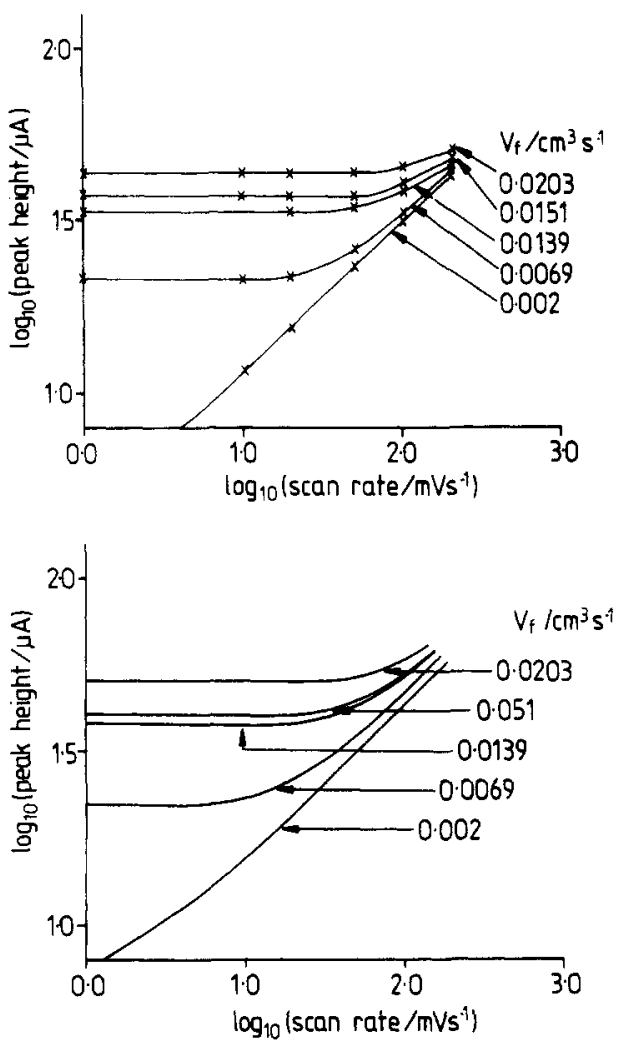

Figure 5. (a, top) Plot of $\log$ (peak current) vs $\log$ (scan rate) constructed from linear sweep voltammograms. Other conditions as Figure $4 \mathrm{~b}$. (b, bottom) Corresponding computed behavior deduced from the theory presented in the text.

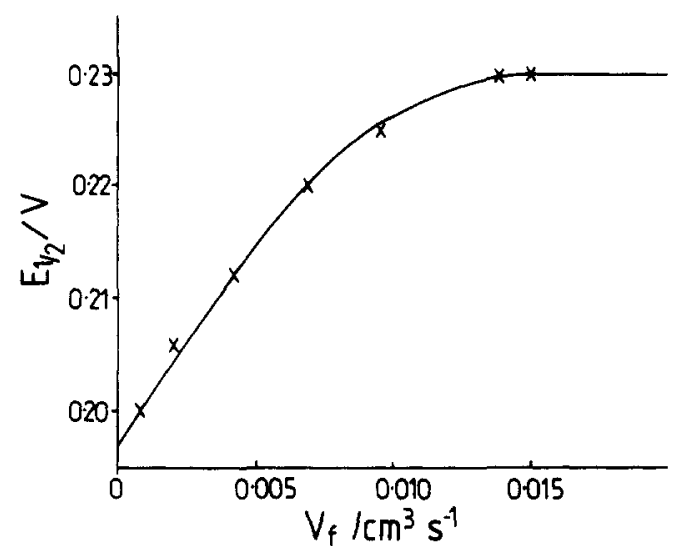

Figure 6. Plot of half-wave potential vs flow rate determined at $1 \mathrm{mV}$ $\mathrm{s}^{-1}$ scan rate; the other conditions are as for Figure 4 .

and $v_{z}$ given in ref 12 the definition of the following dimensionless variables

$$
\begin{aligned}
& \xi=(r / R)^{9 / 8} \\
& \chi=A z / r^{7 / 8}
\end{aligned}
$$

and that of $r$ (eq 7) leads to the following normalized equation:

$$
\xi^{14 / 9} \frac{\partial[\mathrm{A}]}{\partial \tau}=\frac{\partial^{2}[\mathrm{~A}]}{\partial \chi^{2}}-\chi \frac{\partial[\mathrm{A}]}{\partial \xi}
$$

Then on considering eq A 3 together with the boundary conditions (2), (3), (4), and (5) it follows that the current transient when normalized to the steady-state limiting current, $I_{\text {lim }}$, and plotted against $\theta$ (or $\ln \theta$, as in Figure 3 ) should be a unique function of the parameter $\sigma$.

Registry No. Ferrocyanide, 13408-63-4.

\section{References and Notes}

(1) Glauert, M. B. J. Fluid Mech. 1956, 1, 625. 37 .

(2) Aoki, K.; Tokuda, K.; Matsuda, H.J. Electroanal. Chem. 1986, 206, 
(3) Albery, W. J.; Brett, C. M. A. J. Electroanal. Chem. 1983, 148, 211.

(4) Yamada, J.; Matsuda, H. J. Electroanal. Chem. 1973, 44, 189.

(5) Albery, W. J. J. Electroanal. Chem. 1985, 191, 1.

(6) Gunasingham, H.; Fleet, B. Anal. Chem. 1983, 55, 1409.

(7) Schlichting, H. Boundary Layer Theory; Pergamon Press: London, 1955; pp 73-75. 51.

(8) Nanzer, J.; Donizeau, A.; Couret, F. J. Appl. Electrochem. 1984, I4,

(9) Chin, D. T.; Chandran, R. R. J. Electrochem. Soc. 1981, 128, 1904.

(10) Chin, D. T.; Tsang, C. H. J. Electrochem. Soc. 1978, 125, 1461. 345 .
(12) Compton, R. G.; Greaves, C. R.; Waller, A. M. J. Appl. Electrochem. $1990,20,575$.

(13) Gunasingham, H.; Fleet, B. Electroanal. Chem. 1989, 16, 89

(14) Albery, W. J.; Brett, C. M. A. J. Electroanal. Chem. 1983, 148, 201.

(15) Compton, R. G.; Pilkington, M. B. G.; Stearn, G. M. J. Chem. Soc., Faraday Trans. 1 1988, 84, 2155.

(16) Compton, R. F.; Fisher, A. C.; Tyley, G. P. J. Appl. Electrochem. 1990, $20,912$.

(17) Bard, A. J.; Faulkner, L. R. Electrochemical Methods; John Wiley: New York, 1980; 218. 2385 .

\title{
Permanganate Oscillators with Keto Dicarboxyllc Acids
}

\author{
Milan Melicherčíik, Marta Mrákavovă, Arpád Nagy, Anna Olexovā, and Ľudovít Treind* \\ Department of Physical Chemistry, Comenius University, 84215 Bratislava, Czechoslovakia \\ (Received: October 15, 1991; In Final Form: May 26, 1992)
}

\begin{abstract}
A new group of chemical oscillators is reported based on the reduction of $\mathrm{MnO}_{4}^{-}$by ketomalonic, oxalacetic or $\alpha$-ketoglutaric acid in a continuously stirred tank reactor (CSTR). Conditions are described under which a kinetic bistability between an oxidized steady state and a reduced steady state can be observed and under which sustained oscillations occur.
\end{abstract}

\section{Introduction}

The recently discovered permanganate oscillators ${ }^{1-7}$ constitute a new family of chemical oscillators based on transition-metal chemistry. All members of the family consist of permanganate as the oxidant, a reducing substrate $\left(\mathrm{H}_{2} \mathrm{O}_{2}\right.$, ninhydrin, $\mathrm{S}_{2} \mathrm{O}_{3}{ }^{2-}$, $\mathrm{S}^{2-}, \mathrm{SO}_{3}{ }^{2-}, \mathrm{Mn}^{2+}, \mathrm{NH}_{2} \mathrm{OH}, \mathrm{AsO}_{3}{ }^{3-}$, thiocyanate, thiourea, $\mathrm{L}-$ ascorbic acid), and phosphate ion to stabilize the colloidal form of $\mathrm{MnO}_{2}$. A manganese oscillator based on the oxidation of $\mathrm{Mn}^{2+}$ ions by periodate in a continuously stirred tank reactor (CSTR) revealed only very recently also belongs to this family of transition-metal oscillators and might represent a new branch of this family. ${ }^{8,9}$

Since the mechanism of permanganate oscillators is not understood so far, further studies are needed on both branches of transition-metal oscillators.

Therefore, we tried to find even more permanganate oscillators, and the following study might start a new subgroup. It deals with permanganate oscillators based on the oxidation of keto dicarboxylic acids, i.e. ketomalonic (a), oxalacetic (b), and $\alpha$-ketoglutaric acids (c), by $\mathrm{MnO}_{4}^{-}$. We believe that the study of this<smiles>O=C(O)C(=O)O</smiles><smiles>O=C(O)CC(=O)O</smiles><smiles>O=C(O)CCC(=O)C(=O)O</smiles>

subgroup should bring a new insight into the mechanism of permanganate-based oscillators.

\section{Experimental Section}

Materials. Stock solutions of the components besides ketomalonic acid were prepared from commercially available $\mathrm{KMnO}_{4}$

(p.a., Lachema, Brno, Czechoslovakia), $\mathrm{H}_{3} \mathrm{PO}_{4}$ (p.a., Lachema), $\alpha$-ketoglutaric acid (p.a., Loba, Switzerland), and oxalacetic acid (p.a., Reanal, Budapest, Hungary). Ketomalonic acid was prepared by oxidation of saccharose by the procedure of Chattaway and Harris ${ }^{10}$ and was isolated as a bisodium salt that was used for the preparation of stock solutions. A stock solution of $\mathrm{KMnO}_{4}$ was prepared by the procedure of Kolthoff, Belcher, Stenger, and Matsuyama. ${ }^{11}$ The concentration of $\mathrm{H}_{3} \mathrm{PO}_{4}$ was checked alcalimetrically. Dilutions were made with bidistilled water.

Apparatus. We used glass CSTRs ${ }^{12}(27.6 \mathrm{~mL})$ with a mantle connected to a TB 150 ultrathermostat (Medingen) and monitored the oscillatory reaction voltamperometrically (Polarograph LP 7, Laboratorni Prístroje, Prague, Czechoslovakia) by means of a static platinum electrode in a flowing, well-stirred solution. The reaction systems were stirred by a mixing propeller driven by a motor connected to a stabilized voltage supply. Our reactors were equipped with propellers with a stirring frequency of $2250 \mathrm{~min}^{-1}$.

In a closed system the reaction was followed spectrophotometrically using a spectrophotometer (SPECORD M 40 Zeiss, Jena, Germany) with a thermostated $1-\mathrm{cm}$ quartz cuvette with a magnetic stirrer.

The experimental procedure was the same as that in earlier work. ${ }^{1-3,6,9}$

\section{Results and Discussion}

The reduction of $\mathrm{MnO}_{4}^{-}$ions by ketomalonic, oxalacetic, or $\alpha$-ketoglutaric acid in a solution of phosphoric acid proceeds autocatalytically (Figure 1). The course of these autocatalytic reactions and their inflection times depend on the concentrations of both reactants, the concentration of $\mathrm{H}_{2} \mathrm{PO}_{4}^{-}$ions, and $\mathrm{pH}$. The absorbance measured at the wavenumber of $525 \mathrm{~nm}$ (corresponding to $\mathrm{MnO}_{4}{ }^{-}$ions) decreases with time in a sigmoidal way.

We attempted to estimate the region of kinetic bistability by analysis of a sigmoidal curve, using the procedure of Epstein. ${ }^{13}$ For example, if we presume $\mathrm{Mn}^{2+}$ to be the autocatalytic species, we can plot the dependence of reaction rate on the increasing concentration of $\mathrm{Mn}^{2+}$ ions that is calculated from the decrease of $\mathrm{MnO}_{4}^{-}$with an excess of ketomalonic acid. From the minimal and maximal values of flow rates, whose straight lines have three intersects with the curve, a flow rate interval that might correspond to kinetic bistability can be estimated. So we have approached the conditions, and finally we have found the conditions under 